Article

\title{
Near Infrared Spectroscopy Calibration for Wood Chemistry: Which Chemometric Technique Is Best for Prediction and Interpretation?
}

\author{
Brian K. Via ${ }^{1{ }_{*}}$, Chengfeng Zhou ${ }^{1,2}$, Gifty Acquah ${ }^{1}$, Wei Jiang ${ }^{1,3}$ and Lori Eckhardt ${ }^{4}$ \\ 1 Forest Products Development Center, School of Forestry and Wildlife Sciences, Auburn University, \\ 520 Devall Dr., Auburn, AL 36849, USA; E-Mails: czz0024@auburn.edu (C.Z.); \\ gea0002@tigermail.auburn.edu (G.A.); weijiangqd@gmail.com (W.J.) \\ 2 Center for Bioenergy and Bioproducts, Biosystems Engineering, Auburn University, \\ 520 Devall Dr., Auburn, AL 36849, USA \\ 3 College of Textiles, Qingdao University, 308 Ningxia Road, Qingdao 266071, China \\ 4 Forest Health Dynamics Laboratory, School of Forestry and Wildlife Sciences, Auburn University, \\ 602 Duncan Drive, Suite 3301. Auburn, AL 36849, USA; E-Mail: eckhalg@auburn.edu \\ * Author to whom correspondence should be addressed; E-Mail: bkv0003@auburn.edu; \\ Tel.: +1-334-844-1088; Fax: +1-334-844-1084.
}

Received: 29 May 2014; in revised form: 12 July 2014 / Accepted: 21 July 2014 /

Published: 25 July 2014

\begin{abstract}
This paper addresses the precision in factor loadings during partial least squares (PLS) and principal components regression (PCR) of wood chemistry content from near infrared reflectance (NIR) spectra. The precision of the loadings is considered important because these estimates are often utilized to interpret chemometric models or selection of meaningful wavenumbers. Standard laboratory chemistry methods were employed on a mixed genus/species hardwood sample set. PLS and PCR, before and after 1st derivative pretreatment, was utilized for model building and loadings investigation. As demonstrated by others, PLS was found to provide better predictive diagnostics. However, PCR exhibited a more precise estimate of loading peaks which makes PCR better for interpretation. Application of the 1st derivative appeared to assist in improving both PCR and PLS loading precision, but due to the small sample size, the two chemometric methods could not be compared statistically. This work is important because to date most research works have committed to PLS because it yields better predictive performance. But this research suggests there is a tradeoff between better prediction and model interpretation. Future work is needed to compare PLS and PCR for a suite of spectral pretreatment techniques.
\end{abstract}


Keywords: NIR; chemometric; PLS; PCR; regression; loading; coefficient; error; wood chemistry

\section{Introduction}

Near infrared spectroscopy (NIR) is becoming increasingly important for the rapid characterization of wood tissue chemistry. It is rapid and sometimes non-destructive if no grinding is required prior to analysis. In forests and materials derived thereof, NIR has been utilized to predict lignin [1], cellulose [2], hemicellulose [3], extractives [4], cellulose crystallinity [5], p-hydroxyphenyl (H)-, guaiacyl (G)-, and syringyl (S)-based lignin quality [6]. Secondary traits that depend on or correlate to the underlying wood chemistry have also been modeled with NIR spectroscopy including microfibril angle [7], tracheid morphology [8], mechanical properties [9], Kraft pulp yield [10], density [11], shrinkage behavior [12], moisture content [13], sapwood:heartwood ratio [14], and compression wood [15]. Assessment of these type of traits have been used to evaluate forest materials in genetic breeding trials [16,17], silviculture [18], forest products [19], pulp and paper [20], heat treatment [21], and bioenergy [22]. In most cases, the predictive capacity of the NIR model has been the focus of discussion with partial least squares (PLS) working better than principal components regression (PCR) for improved $r^{2}$ and other predictive diagnostics. However, more and more scientists are using the coefficients/loadings within the models to interpret the relationship between wood chemistry functional groups and key traits including tensile strength [23,24], bending [11,25], calorific content [26], among others. But currently, it is unknown if PLS loading plots are statistically similar to that obtained from PCR. In the social sciences discipline, they have cautioned that PLS can be inferior for interpretation [27] while others have warned that shifts in loading location can occur in both PLS and PCR causing error during band assignment and consequent interpretation [28].

During prediction, investigators use these PLS or PCR loadings to assign specific wavenumbers to a chemical compound by assessment of the coefficients (loadings) of statistically significant principal components (PCs), but there may be random error associated with the estimation of these coefficients resulting in some level of uncertainty with either PCR or PLS. It is thought that these errors in PCR could be further inflated during PLS since the location of the "peaks" (coefficients of high and low local values at a given wavenumber) could shift when simultaneously adjusting the $\mathrm{X}$ and $\mathrm{Y}$ matrix for improved prediction. Any shift in these peaks would result in a wavenumber selection that would be slightly different than the real population value. It is thus important to investigate the precision and accuracy of the location of these peak loadings during modeling.

The first derivative has been shown to reduce the severity of the covariance of these adjacent wavelengths [29] in native spectra and it is hypothesized that such a pretreatment would improve the precision of the coefficients/loadings during modeling. But since the first derivative creates a new peak at the location where the slope was the maximum on the raw spectra, then the accuracy of these peaks will be compromised and thus application of the first derivative may improve the precision but lower the accuracy. 
PCR and PLS regression are two multivariate techniques that are usually necessary to overcome the strong covariance in light absorbance between adjacent wavenumbers within a small region of the spectra which inflate the coefficients of the standard multiple linear regression equation [30]. The basic equations for PCR has been described elsewhere [31,32] and is a data reduction-multiple linear regression tool in which significant principal components (PC) are regressed against the dependent variable to construct calibration models of the chemical constituent. The coefficients (loadings) of a specific principal component are then used as weights to express the relative level of influence the original absorbance at that wavenumber has on the overall PC variance and consequently the chemical constituent associated with that PC. These coefficients are assumed to be continuous in nature and a smooth line is used to connect the coefficients resulting in an ability to identify local maximum and minimum loadings in the form of "peaks". These coefficients are then used for interpretation, spectra reduction and remodeling, or in some cases even specific band assignment. The association of functional groups with specific wavenumbers can be made by referencing the literature [28] or regressing it against the chemical component of interest.

One potential weakness of PCR, at least for precision during prediction, is that the PC are developed only from the $\mathrm{X}$ data matrix and there is no consideration for the $\mathrm{Y}$ matrix until the PC are regressed against $Y$. The solution to this problem was the development of PLS regression in which the covariance between $\mathrm{X}$ and $\mathrm{Y}$ is taken into account during PC development [33]. This results in a slightly different data matrix that improves the covariance between the $\mathrm{X}$ and $\mathrm{Y}$ data matrix [33] and results in a higher $\mathrm{r}^{2}$ for the calibration model. But it is postulated that an improvement in covariance between the $\mathrm{X}$ and $\mathrm{Y}$ data matrix will result in a shift in the coefficient location (wavenumber) resulting in inflated error in "peak" location and consequent error during wavenumber selection, interpretation, or band assignment. Conversely, PCR may be a better tool for interpretation/explanation of the model [27] because PLS creates parameter estimates that maximize the covariance between the $\mathrm{X}$ and Y matrix. Thus PLS is more focused on prediction [27] while PCR may better preserve the original X-matrix structure resulting in better model interpretation.

As mentioned earlier, in the forestry and forest materials sector, research has increasing to interpret the coefficients relation to key functional groups and/or the underlying wood chemistry responsible for the response in the $\mathrm{Y}$ variable. Even more work has been done evaluating the predictive capacity of NIR. For example, NIR was used to quantify the patterns of extractives and klason lignin content both radially and longitudinally within 10 Pinus palustris trees [34]. The spectral measurements on these trees were obtained from solid wood surfaces and then related to the wood chemistry. But later it was found that there was supplementary error during prediction when solid wood was used because the tangential, radial, and longitudinal surfaces yields different absorbance patterns during spectra collection [35,36]. Likewise, the radial face was used during the prediction of lignin and monosaccharides which helped to control the predictive error [37]. These technical issues are important because it determines the precision and accuracy of the NIR model to characterize tree tissue chemistry which can impact wood quality based issues [38-41].

Grinding of plant tissues has proven useful during the reduction of prediction error while improving model robustness. For instance, solid wood was ground to 20, 40, and 80 mesh to see if model precision and consequent $\mathrm{r}^{2}$ could be improved [42]. It was found that predicted lignin content exhibited an $r^{2} \approx 0.6$ when the spectra was acquired from the solid wood, increased to an $r^{2} \approx 0.9$ at $20-40$ mesh, 
and increased to an $\mathrm{r}^{2}=0.96-0.99$ for 80 mesh. Grinding was also recommended for Pinus taeda which improved predictions of whole tree properties [43]. For other plant materials such as ramie, grinding was also necessary to achieve stronger calibrations of lignin and cellulose [44]. But in all of these cases, the emphasis has been on increasing the predictive $r^{2}$ or to reduce the predictive error of the Y matrix. To do this, investigators have often chosen PLS over PCR because many studies have demonstrated PLS to have higher predictive capacity. For illustration, lower predictive errors were achieved and with fewer factors when PLS was compared to PCR for the prediction of ash and char content [45]. When visible spectral data was applied to pulp samples, PLS also proved to be slightly more accurate once the optimal number of factors was determined [46]. Similar findings were established when ATR-FTIR was used to predict the delignification of lignin due to rotting fungi [47]. PLS was found to work better than PCR for both NIR and ATR-FTIR for the monitoring of the proximate analysis and heating value of torrefied switchgrass (Panicum virgatum), Pinus taeda, and Liquidambar styraciflua [31].

The primary objective of this paper was to investigate whether the PLS method introduces additional error in the loading plot, when compared to PCR, due to shifts in the loading peaks that might occur during the process of optimizing the covariance between the $\mathrm{X}$ and $\mathrm{Y}$ data matrix. As such, the alternative hypothesis $\left(h_{a}\right)$ for this experiment is that the loadings/coefficients in the PLS will decrease in precision and consequently increase in variance. To test this hypothesis, the location of the local peak loading, obtained through PLS and PCR coefficient plots, will be subtracted from the best representative band assignments as obtained from the literature:

$$
\mathrm{C}-\mathrm{BA}_{\mathrm{L}}=\mathrm{R}
$$

where $\mathrm{C}$ represents wavenumber obtained through PLS or PCR analysis; $\mathrm{BA}_{\mathrm{L}}$ is the best representative band assignment obtained from the literature; and $\mathrm{R}$ represents the Residual between $\mathrm{C}$ and $\mathrm{BA}_{\mathrm{L}}$. Then the variance of the residuals will be further tested under the following hypothesis constructs:

$$
\begin{aligned}
& \mathrm{H}_{0}: \sigma_{\text {PLS-R }}^{2}=\sigma_{\text {PCR-R }}^{2} \\
& \mathrm{H}_{\mathrm{a}}: \sigma_{\text {PLS-R }}^{2}>\sigma_{\text {PCR-R }}^{2}
\end{aligned}
$$

where $\sigma^{2}$ represents the variance of R obtained from PLS or PCR models. Differences for variance between model loadings will be tested by the F-Test of $\mathrm{R}[30]$.

\section{Experimental Section}

\subsection{Sample Preparation}

All samples were collected from recently harvested hardwood trees. There were four different genera and 37 samples including four Eucalyptus, nine cotton wood, 12 aspen and 12 poplar. First, the wood samples were planed down to $3 \mathrm{~mm}$ thick wood chips and then stored for 2 weeks at $24 \pm 1.5{ }^{\circ} \mathrm{C}$ and $45 \% \pm 5 \%$ relative humidity. Two weeks was enough time to reach equilibrium with the environment; i.e., the weight of the biomass no longer decreased with time. Then $50 \mathrm{~g}$ of air dried samples were ground to 40 mesh using a Willey mill and then $20 \mathrm{~g}$ of 40 mesh samples were further 
ground to 80 mesh. The 40 mesh samples were used for wood chemistry analysis (wood chemistry section) and the 80 mesh samples were used for FT-NIR spectra collection (FT-NIR acquisition).

\subsection{Wood Chemistry}

The extractives, lignin and monosaccharide contents of 37 samples were measured following National Renewable Energy Laboratory (NREL) standards [48,49]. The cellulose, hemicellulose and holocellulose contents were also measured by traditional wet chemistry analysis. As shown in Figure 1, $150 \mathrm{~mL}$ acetone was used to extract $5 \mathrm{~g}$ of sample for $6 \mathrm{~h}$ to get acetone based extractives. After extractives removal, the extractive free sample was separated into 2 batches.

Figure 1. Chemical content $(\%, w / w)$ measured both in the laboratory and that predicted by NIR for validation samples.

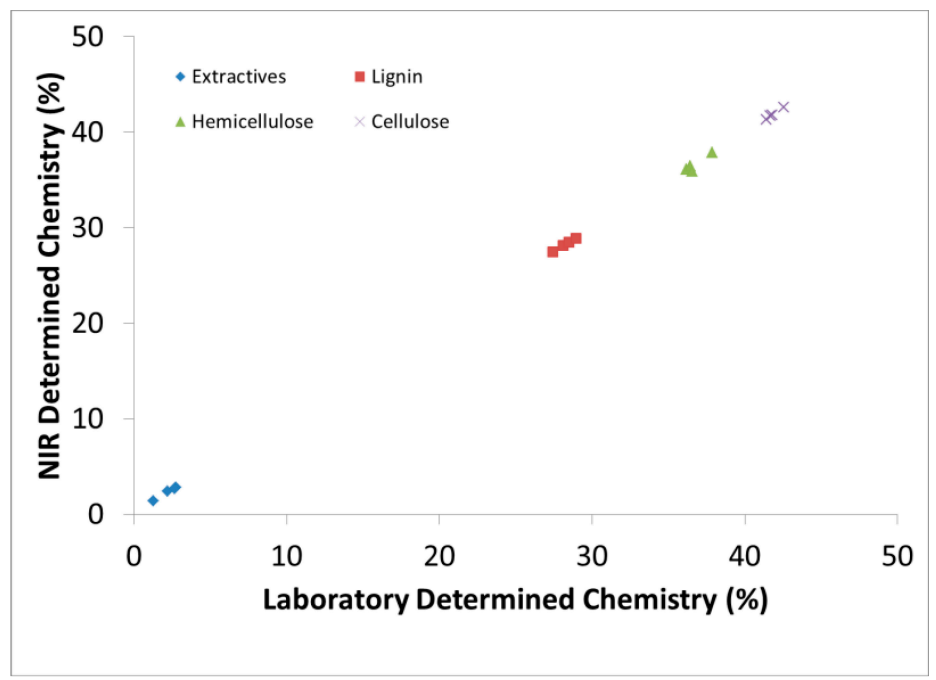

Batch 1: A $72 \%(\mathrm{w} / \mathrm{w})$ sulfuric acid treatment at $30{ }^{\circ} \mathrm{C}$ for $2 \mathrm{~h}$ was used to prehydrolyze the extractive free sample. The solution was then diluted to $4 \%$ sulfuric acid with distilled water, sealed in a bottle and placed in an autoclave for $1 \mathrm{~h}$ at $121^{\circ} \mathrm{C}$, and then the residual from the bottle was filtered and oven dried to measur lignin content. The extractives and lignin contents were measured by gravimetric analysis. To determine the monosaccharide composition, an HPLC (Shimadzu LC-20A), equipped with an Aminex $87 \mathrm{P}$ column and differential refractive index detector and the sugar solution was analyzed. Holocellulose content (Holo-HPLC) was calculated as the sum of all the monosaccharides contents.

Batch 2: Delignification treatments were conducted to determine the holocellulose content. The delignification procedure was as follows. First, $2 \mathrm{~g}$ was weighed separately and placed into conical flasks $(500 \mathrm{~mL})$ with $320 \mathrm{~mL}$ of distilled water in each flask. Second, the flasks were placed into a water bath $\left(75^{\circ} \mathrm{C}\right)$ and the samples were placed into the flasks. Then $1 \mathrm{~mL}$ of acetic acid and $20 \mathrm{~mL}$ $15 \%(\mathrm{w} / \mathrm{w})$ sodium chlorite were added into each flask on a 1-h cycle for $4 \mathrm{~h}$. After $4 \mathrm{~h}$, the residues were filtered with filter paper and then oven dried for $3 \mathrm{~h}$ to test the holocellulose content. Then, $1.5 \mathrm{~g}$ of oven dried holocellulose was placed into a $250 \mathrm{~mL}$ conical flask. One-hundred $\mathrm{mL}$ of $17.5 \%$ sodium hydroxide was stirred into the flask and the air was replaced with nitrogen and the flask was immediately sealed with aluminum foil. The flask was then placed in a water bath at $20{ }^{\circ} \mathrm{C}$ and stirred occasionally 
until the reaction was complete. The solution was then filtered through a pre-weighed filter paper and washed with $500 \mathrm{~mL}$ of distilled water. The sample was then oven dried at $105{ }^{\circ} \mathrm{C}$ for $12 \mathrm{~h}$ and weighed. The residue was determined as cellulose and the hemicellulose content was considered to be the difference in holocellulose and cellulose.

When conducting wet chemistry, all samples were air dried and tested for moisture content to calculate the dry weight of the original samples and such that moisture was not included as weight during gravimetric determination of the wood polymers. All experiments were performed in duplicate. All chemicals were purchased from VWR Company (Atlanta, GA, USA), and were analytically or chromatographically pure.

\subsection{FT-NIR Acquisition}

Samples were oven dried for $12 \mathrm{~h}$ and then placed into a dessicator to maintain near ovendry conditions but remove the effect of changing temperature on spectra fluctuation [50]. For each sample, the wood powder was placed on the FT-NIR machine to avoid packing and the reflectance spectra were collected on a window that was $8 \mathrm{~mm}$ in diameter. A PerkinElmer (Waltham, MA, USA) spectrum 400 FT-NIR spectrometer was utilized for spectra collection. The spectra covered the range of $10,000-4,000 \mathrm{~cm}^{-1}$ at a spectral resolution of $4 \mathrm{~cm}^{-1}$. Each spectrum was collected from an average of 32 scans and no zero filling. It should be noted that no smoothing was applied to the raw spectra because after 16 scans, there was no difference in the spectra before or after smoothing. Thirty-two scans were thus chosen for superior precision. Baseline analysis was also run on the raw spectra but there was no change in loading peaks so the raw spectra were analyzed with no pretreatment.

\subsection{Chemometric Analysis}

PCR and PLS modules in Spectrum Quant + software was used for model construction. Models were executed on the unprocessed spectra (raw) and first derivative (FD). The FD was computed prior to PCR or PLS modeling and was calculated with the Savitzky-Golay approach (2nd order polynomial with 25 points). Thirty-one samples were used to construct models and 6 samples were used for validation. Because of the small sample size, cross validation on all 37 samples (leave one out 37 times) was also ran to ensure similar results and insulate against one data point (out of five) biasing or inflating parameter estimates during validation. While the population for calibration and validation were randomly selected, the distribution of the data was checked to ensure a similar mean and range between the two populations. The predictive performance of the models in this paper was evaluated by several standards, including the coefficient of determination $\left(\mathrm{r}^{2}\right)$, root mean square error of calibration (RMSEC), and root mean square error of prediction (RMSEP) [30]. The residual predictive deviation (RPD) was also measured to understand whether models could potentially be used in real measurement systems, screening, or just for interpretation purposes [42].

For PCR coefficient/loading plots, the most statistically significant PC to relate to the chemical constituent during multivariate modeling was utilized. The coefficients ( $y$-axis) were connected via a smooth line in Origin software and then plotted against the wavenumbers ( $x$-axis). For PLS, the regression coefficients plot was computed which represents the relationship between all of the 
absorbance (entire wavenumber range) and the specific chemical constituent of interest. The peak locations were then chosen and compared to wavenumbers chosen a-priori from the literature.

\section{Results and Discussion}

\subsection{Predictive Diagnostics}

Table 1 demonstrates the summary statistics for the best predictive models. In every case, PLS outperformed PCR in predictive diagnostics (not shown). Application of the 1st derivative resulted in better calibration models than when the raw spectrum was utilized. Preprocessing with the 1st derivative demonstrated better prediction based on the higher $\mathrm{r}^{2}$, a lower RMSEP, and a higher RPD. The sometimes drastic improvement in prediction with the 1st derivative was perhaps due to the presence of a baseline shift which can impact the computation of the 1st PC. These differences in model performance before and after derivative pretreatment was not expected. It was our pre-conjecture that grinding to a fine powder ( 80 mesh) would minimize any inherent solid wood density variations between samples which can cause baseline shifts in the spectra [42]. Similar improvements were observed during the prediction of wood cellulose crystallinity when the 1st derivative was applied and they also milled their samples [5]. Others have supported that baseline shifts and bias is often unavoidable in NIR spectra due to subtle differences in path length and differences in light scattering between samples [51]. It was also possible that some particles settled resulting in increased variation in bulk density although every effort was made to minimize this effect. It was also noticed that for smaller sample sizes such as in this and other studies from our laboratory [42], pretreatments were more necessary for calibration improvement than when larger data sets were employed [31].

Table 1. Calibration and predictive results of NIR based multivariate (PLS) models.

\begin{tabular}{cccccccc}
\hline \multirow{2}{*}{ Chemistry } & \multicolumn{3}{c}{ Raw Spectra } & & \multicolumn{3}{c}{ First Derivative } \\
\cline { 2 - 4 } \cline { 6 - 8 } & $\mathbf{r}^{\mathbf{2}}$ & RMSEP & RPD & & $\mathbf{r}^{\mathbf{2}}$ & RMSEP & RPD \\
\hline Extractives & 40.7 & 1.18 & 1.21 & & 85.0 & 0.98 & 1.45 \\
Lignin & 82.6 & 1.35 & 1.78 & & 90.4 & 1.12 & 2.15 \\
Cellulose & 37.6 & 2.10 & 1.00 & & 81.0 & 1.03 & 2.04 \\
Hemicellulose & 41.9 & 3.17 & 1.23 & & 93.5 & 1.43 & 2.72 \\
\hline
\end{tabular}

Figure 1 demonstrates the capability to predict new samples based on calibration models and how one can simultaneously predict several wood chemical constituents from one spectral measurement. The predictive statistics in Table 1, however, were obtained through cross validation (leave one out method). We chose to use both methods to demonstrate the validity of the models but focused more on the cross validation method during model selection which has been shown to be better for small data sets [30].

\subsection{Assessment of Loading Plots}

For most loading plots, application of the 1st derivative resulted in more similar plots between PLS and PCR. For extractives prediction, the loading patterns were very dissimilar for the two modeling methods when the raw spectra were utilized (Figure 2). 
Figure 2. Coefficients by wavenumber for PCR and PLS for extractives prediction (a) when raw spectra was processed and (b) when a first derivative pretreatment was processed. PC number 9, 1, 5, and 3 were chosen for PCR-raw, PLS-raw, PCR-derivative, and PLS derivative respectively $(\alpha=0.05)$.

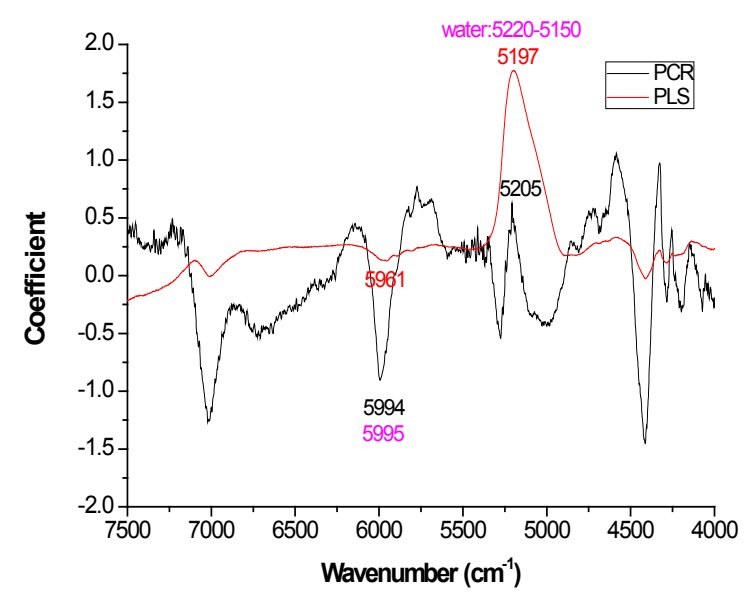

(a)

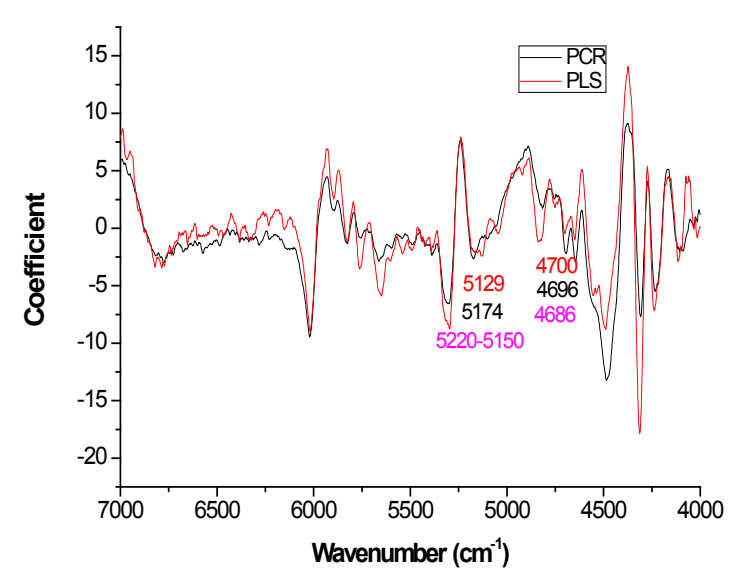

(b)

The absolute magnitude of the coefficients was higher for PCR while PLS exhibited flatter plots with perhaps the only distinctive peak occurring at $5197 \mathrm{~cm}^{-1}$ (Figure 2a). However, when the first derivative was applied, there was no visual difference in coefficient intensity between PCR and PLS (Figure 2b).

Figure 3. Coefficients by wavenumber for PCR and PLS for lignin prediction (a) when raw spectra was processed and (b) when a 1st derivative pretreatment was processed. PC number 9, 4, 5, and 3 were chosen for PCR-raw, PLS-raw, PCR-derivative, and PLS derivative respectively $(\alpha=0.05)$.

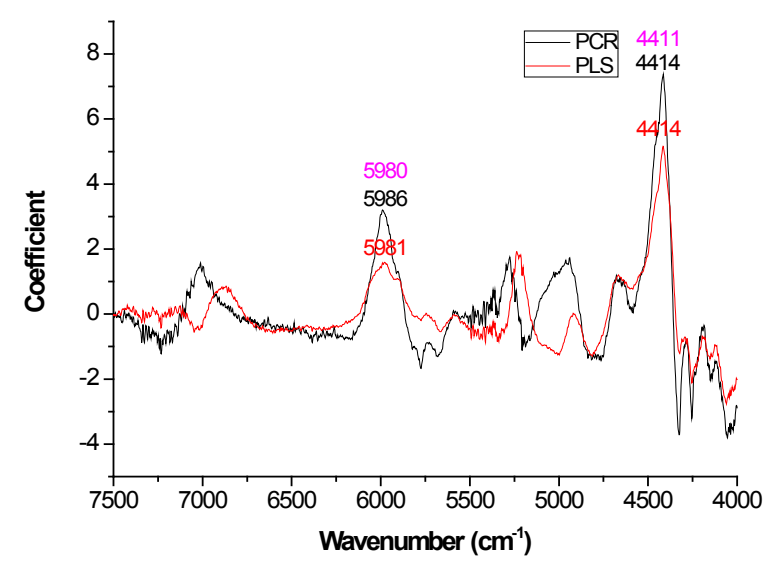

(a)

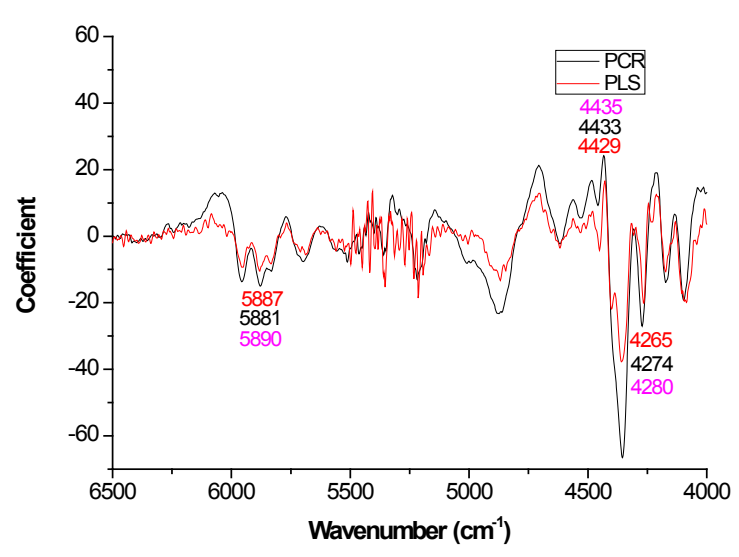

(b) 
Figure 4. Coefficients by wavenumber for PCR and PLS for cellulose prediction (a) when raw spectra was processed and (b) when a 1st derivative pretreatment was processed. PC number 10, 8, 4, and 4 were chosen for PCR-raw, PLS-raw, PCR-derivative, and PLS derivative respectively ( $\alpha=0.05)$.

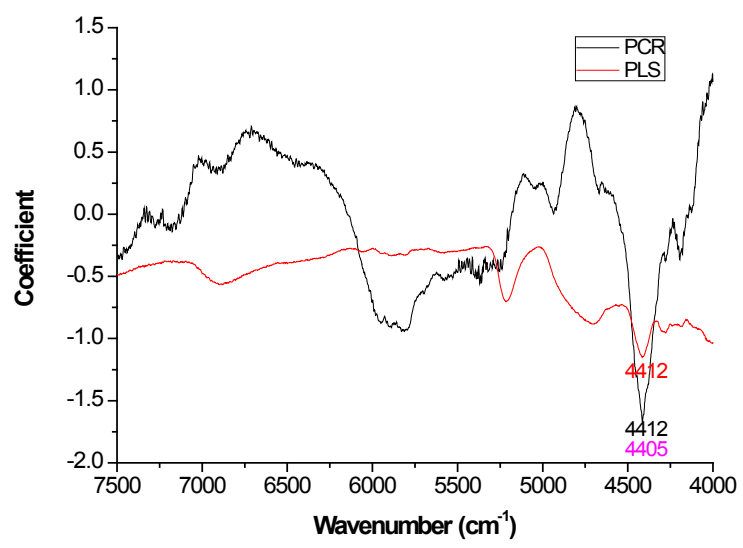

(a)

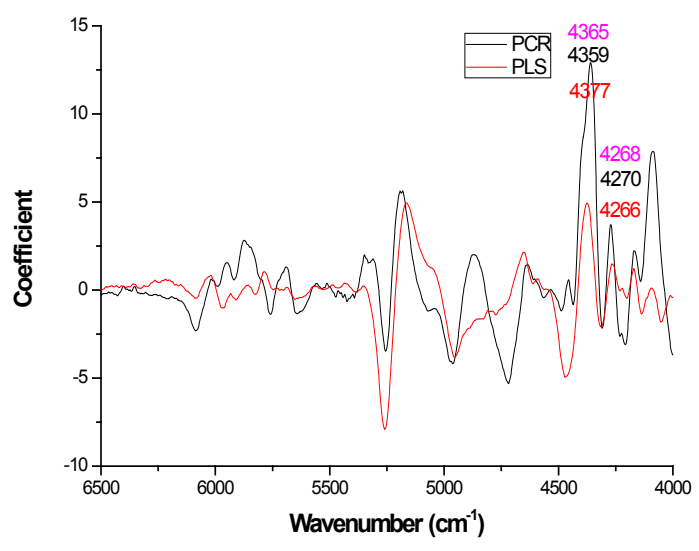

(b)

Figure 5. Coefficients by wavenumber for PCR and PLS for hemicellulose prediction (a) when raw spectra was processed and (b) when a 1st derivative pretreatment was processed. PC number 2, 1, 1, and 1 were chosen for PCR-raw, PLS-raw, PCR-derivative, and PLS derivative respectively $(\alpha=0.05)$.

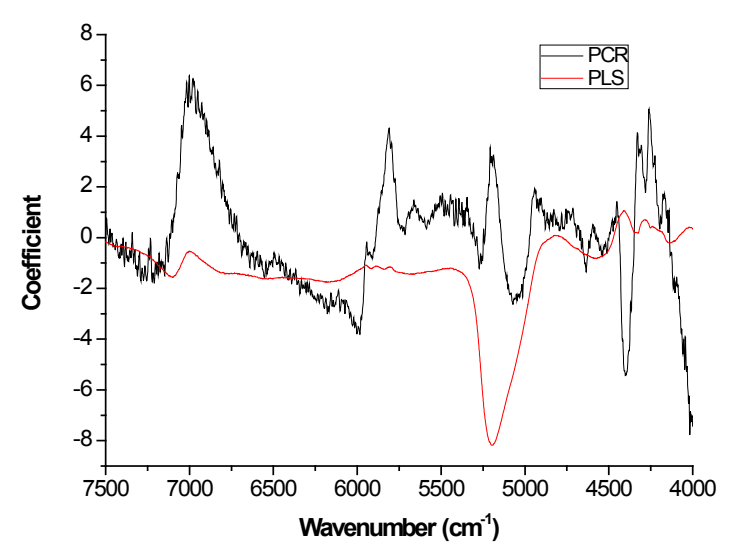

(a)

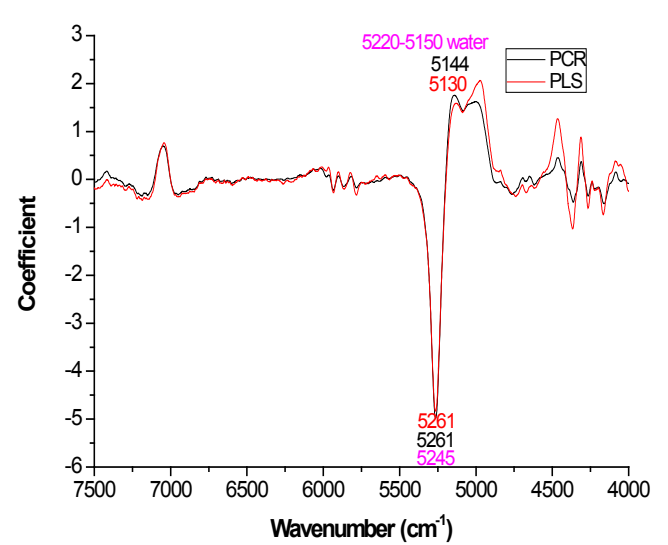

(b)

Mild improvements in PLS coefficient estimates (when compared to PCR) also occurred with the first derivative for lignin but once again PCR coefficients were slightly higher for the raw spectra based models (Figure 3a). For cellulose and hemicellulose, coefficient plots between the two methods became much more similar after first derivative application (Figures 4-5). In other words, the loading plots became more "parallel" or similar in pattern.

The general improvement in PLS coefficient plots with a 1st derivative pretreatment was probably attributable to the removal of the baseline shift that occurs due to physical rather than chemical features of the material and 25 point smoothing. For Pinus palustris, and Pinus spp., it was demonstrated that an increase in solid wood density coincided with a linear increase in absorbance [35,52]. With 
PCR, the first PC will partition the variation due to the baseline shift such that better signals attributable to the underlying chemistry can be resolved through other PC [53].

With careful evaluation, it was also noticeable that there was sometimes a shift in the location of the peak coefficient when going from the native to 1 st derivative based data sets. To illustrate, for the extractives models, the wavenumber at $5197-5205 \mathrm{~cm}^{-1}$ shifted to $5174 \mathrm{~cm}^{-1}$ when the $1 \mathrm{st}$ derivative pretreatment was used. This 20 to $30 \mathrm{~cm}^{-1}$ shift in absolute maximum coefficient could also be seen for lignin (4411 to $4435 \mathrm{~cm}^{-1}$ ) and hemicellulose (5225 to $5245 \mathrm{~cm}^{-1}$ ) (Figures 2 and 4). These errors will be quantified statistically later in the paper (Table 2).

Table 2. Hypothesis testing of Equations (2) and (3) through the F-Test. * means the F-Test was significant with $95 \%$ confidence.

\begin{tabular}{ccc}
\hline & PCR & PLS \\
\hline Mean R & -3.4 & -9.4 \\
Variance & 189 & 700 \\
Standard deviation & 13.7 & 26.5 \\
95\% CI & $-3.4 \pm 7.6$ & $-9.4 \pm 14.7$ \\
Observations & 15 & 15 \\
Degrees of freedom & 14 & 14 \\
F & 0.27 & \\
$\mathrm{P}(\mathrm{F}<\mathrm{f})$ one tail & $0.0099 *$ & \\
\hline
\end{tabular}

For a given local region, this shift in location of the peak coefficient can be explained by the fact that the peak in the first derivative occurs at the same location as an inflection point location in the native spectra. A solution to this problem would be to take the 2 nd derivative which will theoretically fall in the same location while simultaneously removing the baseline shift effect. However, with each derivative applied, the risk of lower signal to noise ratio increases which will have unknown effects on the prediction of the chemistry of future populations. This concept was demonstrated for blue stained tissue in which the confidence intervals for absorption were wildly inflated when transitioning from the 1 st to 2 nd derivative [54]. In that study, application of the 1st derivative maintained statistically similar confidence intervals as that obtained with the native spectra [55].

\subsection{Interpretation of Significant Coefficients and Loadings Error Assessment}

In this study, for the prediction of lignin, the O-H, C-O, C-H stretch and the aromatic skeletal vibrations were important loadings at 4401, 4411, and $4280 \mathrm{~cm}^{-1}$ respectively [28]. For extractives prediction, the C-O and $\mathrm{O}-\mathrm{H}$ bond was important based on loadings at 6913 and $7092 \mathrm{~cm}^{-1}$ [28]. For cellulose prediction, the $\mathrm{C}-\mathrm{H}$ and $\mathrm{CH} 2$ deformation were key functional groups that were important based on loadings at $6307,5814,4405 \mathrm{~cm}^{-1}$ [28]. Hemicellulose quantification yielded $\mathrm{C}-\mathrm{H}$ and $\mathrm{C}=\mathrm{O}$ bond based on loadings at $7410,6003,5236$, and $4686 \mathrm{~cm}^{-1}$.

It should be noted that band assignments given above were those standard to the literature $\left(\mathrm{BA}_{\mathrm{L}}\right)$ and were chosen a priori while the loadings in the models, as anticipated, exhibited some level of error around $\mathrm{BA}_{\mathrm{L}}$. The distribution of error $(\mathrm{R})$ for both PCR and PLS exhibited a skewed pattern while PCR exhibited a distribution closer to normality (Figure 6). Visually, the error appeared to be slightly biased 
to one side of zero, but when a confidence interval test was performed for both PLS and PCR $(\alpha=0.05)$, both overlapped with zero (Table 2). Thus statistically, both methods were still accurate for proper wavenumber selection and assignment. Nevertheless, the R (distribution of error) in PLS was higher in variance and this made it more difficult to determine if the mean differed from zero through confidence interval testing for the number of degrees of freedom available. As Figure 6 demonstrates, it is quite possible that PLS did introduce bias in loading location.

Figure 6. Frequency of R from PLS and PCR loadings of wood chemistry models.

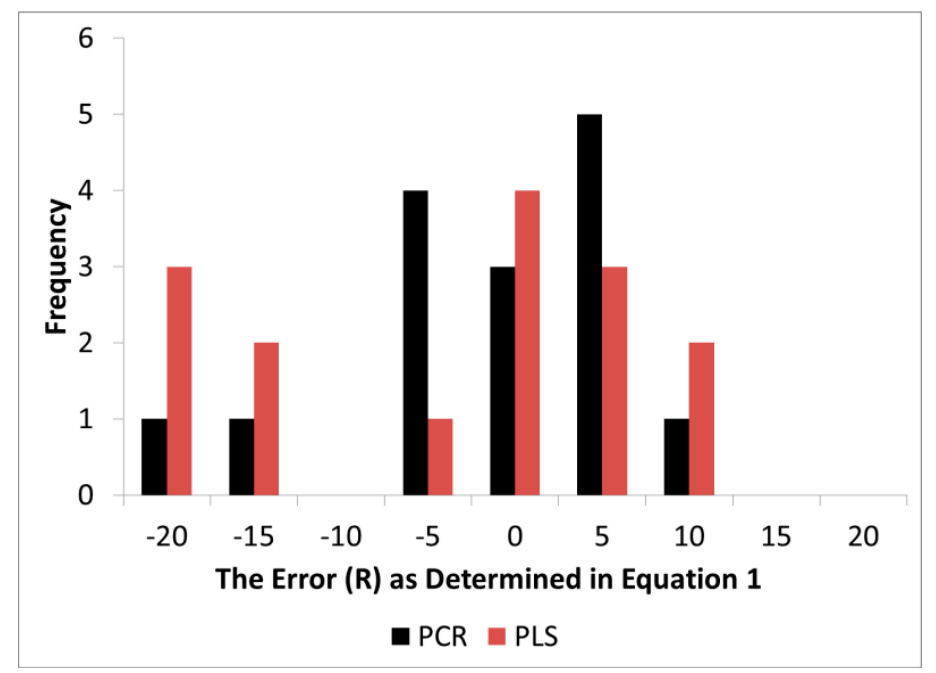

The precision of the location of the peak loading was tested through hypothesis testing. The alternative hypothesis $\mathrm{H}_{0}$ was developed because it was believed that PLS will compromise loadings estimates in order to maximize $\mathrm{X}$ and $\mathrm{Y}$ matrix covariance. An F-Test revealed that the variance in $\mathrm{R}$ for PLS was statistically greater than PCR. This means that while PLS exhibited better prediction of wood chemistry, the error $(\mathrm{R})$ in the loadings estimates increased making wavenumber selection through modeling less certain with PLS.

The precision of PCR for identification of peak location has been adjacently investigated and explained by similar research in the field of 2D correlation spectroscopy in which a perturbation was added to improve the precision of band identification during shift [56]. Two-dimensional correlation spectroscopy and waterfall plots was explored to decipher subtle peak shifts which was a challenge due to overlapping wavenumbers within the local IR region. They explored principal components analysis (PCA) as a supplementary method for monitoring band shift and they were surprised to find this analytical tool to be very sensitive to true maximum peak shift. Their research findings perhaps suggests that the peak variance found in our PCR analysis may be more inherent to sample to sample variation while we think the additional peak variance introduced during PLS analysis (Table 2) for this study was the result of X-matrix modification during X-Y covariance optimization.

Similar to Ryu et al. [56] and this study, another similar finding was present for the analysis of NIR spectra which was acquired from longleaf pine (Pinus palustris) [29]. When the 1st derivative pretreatment was applied and then spectra separated based on stiffness and strength perturbations, lignin and cellulose associated wavelengths were easily separated while hemicellulose was not discernable. But when PC loadings were investigated, they witnessed a significant peak at $2330 \mathrm{~nm}$ 
attributable to the $\mathrm{CH}$ stretch in hemicellulose. In our study, there was not a statistical difference between the error (R) in PCR and PLS after application of the 1st derivative $(\alpha=0.05)$. It is thus thought that the 1st derivative may be a tool to improve loading plot precision; however, most of the degrees of freedom were necessary for testing the original hypothesis that PLS and PCR (in general) differed in error. We thus recommend a separate study in the future to better quantify the improvement in precision with a derivative application. Indeed PCA has recently been shown to be more sensitive to spectra variance for samples with complex reactions or for wood chemistry that possesses similar functional groups. Perhaps that helps to explain it utility as a superior interpretation tool during chemometric modeling such as that employed in this study.

\subsection{Closing Observations}

In closing, the data analysis for this study was the first of its kind, in part, because it took several models (raw and 1st derivative) and multiple wood chemistry traits combined to yield enough degrees of freedom to test for significant differences in targeted loadings between PLS and PCR (Table 2). Unfortunately, the sample size was too low to further test for significant differences in $\mathrm{R}$ for native versus 1st derivative spectra. Since the loadings (Figures 2-5) appeared more stable for 1st derivative based models, future work is necessary to determine if R decreases for PLS after pretreating with the 1 st derivative and this work should be compared to PCR. Other pretreatments or even 2D correlation may also be useful for reduction in $\mathrm{R}$ and should also be tested for in future studies.

\section{Conclusions}

The main purpose of this study was to assess the performance of PLS and PCR for interpretation purposes. In order to do that, we had to quantify the potential error in loading plots and in particular any deviation in the location of the "peaks" from the true population value. It was found that PLS did a better job during prediction while PCR exhibited better precision in identifying the correct loading position and consequently PCR would be better for model interpretation, wavenumber selection, or similar activities. Application of the 1st derivative appeared promising in that the shapes between PCR and PLS loading plots became more similar or "parallel". But future research is necessary to better understand if the 1st derivative or any other pretreatment can yield PLS loading plots with better precision than what was obtained in this study.

This work is important because it suggests that what is best for prediction is not best for model interpretation. Currently, most papers focus on PLS because it does a better job at prediction. But by nature, when the same investigators transition to model interpretation, they may be biased toward PLS because of its superior predictive nature and their prior use of the model. We prescribe that chemometricians consider PCR for their toolbox when performing model interpretation.

\section{Acknowledgments}

This work was most supported by the Agriculture and Food Research Initiative (AFRI) CAP_- "Southeast Partnership for Integrated Biomass Supply Systems"; the Department of Energy grant titled, "High Tonnage Forest Biomass Production Systems from Southern Pine Energy 
Plantations". Other smaller contributions included the Hatch in which these calibration equations will be utilized to partition out feedstocks of high lignin for adhesive production and energy applications, Regions Bank; the National Natural Science Foundation of China (50973048); National Natural Science Foundation of Shandong Province (ZR2012EM002); Special Foundation of "Taishan Scholar" Construction and National Science \& Technology Pillar Program (2012BAD32B06).

\section{Author Contributions}

The work was carried out by the collaboration of all the authors. Brian K. Via conceived the idea, organized the paper, acquired the funding and supervised the work. Chengfeng Zhou carried out the experiments, analyzed the data and interpreted the results. Gifty Acquah and Wei Jiang participated in the experiment and analysis in part. Lori Eckhardt is the co-supervisor. All authors have contributed to the paper.

\section{Conflicts of Interest}

The authors declare no conflict of interest.

\section{References}

1. Ono, K.; Hiraide, M.; Amari, M. Determination of lignin, holocellulose, and organic solvent extractives in fresh leaf, litterfall, and organic material on forest floor using near-infrared reflectance spectroscopy. J. For. Res. 2003, 8, 191-198.

2. McLellan, T.M.; Aber, J.D.; Martin, M.E.; Melillo, J.M.; Nadelhoffer, K.J. Determination of nitrogen, lignin, and cellulose content of decomposing leaf material by near infrared reflectance spectroscopy. Can. J. For. Res. 1991, 21, 1684-1688.

3. Yao, S.; Jiang, Y.-F.; Lu, H.-K.; Su, M. Extending hemicelluloses content calibration of acacia spp using nir to new sites. Spectrosc. Spectr. Anal. 2010, 30, 1206-1209.

4. Gierlinger, N.; Schwanninger, M.; Hinterstoisser, B.; Wimmer, R. Rapid determination of heartwood extractives in larix sp. By means of fourier transform near infrared spectroscopy. J. Near Infrared Spectrosc. 2002, 10, 203-214.

5. Jiang, Z.-H.; Yang, Z.; So, C.-L.; Hse, C.-Y. Rapid prediction of wood crystallinity in pinus elliotii plantation wood by near-infrared spectroscopy. J. Wood Sci. 2007, 53, 449-453.

6. Robinson, A.R.; Mansfield, S.D. Rapid analysis of poplar lignin monomer composition by a streamlined thioacidolysis procedure and near-infrared reflectance-based prediction modeling. Plant J. 2009, 58, 706-714.

7. Schimleck, L.R.; Evans, R. Estimation of microfibril angle of increment cores by near infrared spectroscopy. IAWA J. 2002, 23, 225-234.

8. Jones, P.D.; Schimleck, L.R.; Peter, G.F.; Daniels, R.F.; Clark Iii, A. Non-destructive estimation of pinus taeda 1 tracheid morphological characteristics for samples from a wide range of sites in georgia. Wood Sci. Technol. 2005, 39, 529-545. 
9. Kelley, S.S.; Rials, T.G.; Snell, R.; Groom, L.H.; Sluiter, A. Use of near infrared spectroscopy to measure the chemical and mechanical properties of solid wood. Wood Sci. Technol. 2004, 38, $257-276$.

10. Downes, G.M.; Meder, R.; Hicks, C.; Ebdon, N. Developing and evaluating a multisite and multispecies nir calibration for the prediction of kraft pulp yield in eucalypts. South. For. J. For. Sci. 2009, 71, 155-164.

11. Hoffmeyer, P.; Pedersen, J.G. Evaluation of density and strength of norway spruce wood by near infrared reflectance spectroscopy. Holz als Roh-und Werkst. 1995, 53, 165-170.

12. Baillères, H.; Davrieux, F.; Ham-Pichavant, F. Near infrared analysis as a tool for rapid screening of some major wood characteristics in a eucalyptus breeding program. Ann. For. Sci. 2002, 59, 479-490.

13. Leblon, B.; Adedipe, O.; Hans, G.; Haddadi, A.; Tsuchikawa, S.; Burger, J.; Stirling, R.; Pirouz, Z.; Groves, K.; Nader, J.; et al. A review of near-infrared spectroscopy for monitoring moisture content and density of solid wood. For. Chron. 2013, 89, 595-606.

14. Stirling, R. Near-infrared spectroscopy as a potential quality assurance tool for the wood preservation industry. For. Chron. 2013, 89, 654-658.

15. Chen, Q.M.; Hu, Z.; Chang, H.M.; Li, B. Micro analytical methods for determination of compression wood content in loblolly pine. J. Wood Chem. Technol. 2007, 27, 169-178.

16. Raymond, C.A.; Schimleck, L.R. Development of near infrared reflectance analysis calibrations for estimating genetic parameters for cellulose content in eucalyptus globulus. Can. J. For. Res. 2002, 32, 170-176.

17. Zhang, J.; Novaes, E.; Kirst, M.; Peter, G.F. Comparison of pyrolysis mass spectrometry and near infrared spectroscopy for genetic analysis of lignocellulose chemical composition in populus. Forests 2014, 5, 466-481.

18. Sandak, A.; Sandak, J.; Negri, M. Relationship between near-infrared (NIR) spectra and the geographical provenance of timber. Wood Sci. Technol. 2011, 45, 35-48.

19. So, C.; Via, B.; Groom, L.; Schimleck, L.; Shupe, T.; Kelley, S.; Rials, T. Near infrared spectroscopy in the forest products industry. For. Prod. J. 2004, 54, 6-16.

20. Tsuchikawa, S. A review of recent near infrared research for wood and paper. Appl. Spectrosc. Rev. 2007, 42, 43-71.

21. Esteves, B.; Pereira, H. Wood modification by heat treatment: A review. BioResources 2008, 4, 370-404.

22. So, C.-L.; Eberhardt, T.L. Chemical and calorific characterisation of longleaf pine using near infrared spectroscopy. J. Near Infrared Spectrosc. 2010, 18, 417-423.

23. Tsuchikawa, S.; Hirashima, Y.; Sasaki, Y.; Ando, K. Near-infrared spectroscopic study of the physical and mechanical properties of wood with meso-and micro-scale anatomical observation. Appl. Spectrosc. 2005, 59, 86-93.

24. Kohan, N.; Via, B.; Taylor, S. Prediction of strand feedstock mechanical properties with near infrared spectroscopy. Bioresources 2012, 7, 2996-3007.

25. Via, B.; Jiang, W. Nonlinear multivariate modeling of strand mechanical properties with near-infrared spectroscopy. For. Chron. 2013, 89, 621-630. 
26. Lestander, T.A.; Rhén, C. Multivariate nir spectroscopy models for moisture, ash and calorific content in biofuels using bi-orthogonal partial least squares regression. Analyst 2005, 130, 1182-1189.

27. Hair, J.F.; Black, B.; Babin, B.; Anderson, R.E. Multivariate Data Analysis 7th Pearson Prentice Hall. Up. Saddle River NJ 2010; pp. 752-753.

28. Schwanninger, M.; Rodrigues, J.; Fackler, K. A review of band assignments in near infrared spectra of wood and wood components. J. Near Infrared Spectrosc. 2011, 19, 287.

29. Via, B.; So, C.; Shupe, T.; Groom, L.; Wikaira, J. Mechanical response of longleaf pine to variation in microfibril angle, chemistry associated wavelengths, density, and radial position. Compos. Part A Appl. Sci. Manuf. 2009, 40, 60-66.

30. Neter, J.; Wasserman, W.; Kutner, M.H. Applied Linear Statistical Models: Regression analysis, Analysis of Variance, and Experimental Design, 3rd ed.; Irwin: Homewood, IL, USA, 1990; xvi, p. 1181.

31. Via, B.; Adhikari, S.; Taylor, S. Modeling for proximate analysis and heating value of torrefied biomass with vibration spectroscopy. Bioresour. Technol. 2013, 133, 1-8.

32. Cowe, I.A.; McNicol, J.W. The use of principal components in the analysis of near-infrared spectra. Appl. Spectrosc. 1985, 39, 257-266.

33. Brereton, R.G. Applied Chemometrics for Scientists; Wiley: Hoboken, NJ, USA, 2007; pp. 211-214.

34. Via, B.K.; So, C.L.; Groom, L.H.; Shupe, T.F.; Stine, M.; Wikaira, J. Within tree variation of lignin, extractives, and microfibril angle coupled with the theoretical and near infrared modeling of microfibril angle. IAWA J. 2007, 28, 189-209.

35. Via, B.; McDonald, T.; Fulton, J. Nonlinear multivariate modeling of strand density from near-infrared spectra. Wood Sci. Technol. 2012, 46, 1073-1084.

36. Defo, M.; Taylor, A.M.; Bond, B. Determination of moisture content and density of fresh-sawn red oak lumber by near infrared spectroscopy. For. Prod. J. 2007, 57, 68-72.

37. Jones, P.D.; Schimleck, L.R.; Peter, G.F.; Daniels, R.F.; Clark, A., III. Nondestructive estimation of wood chemical composition of sections of radial wood strips by diffuse reflectance near infrared spectroscopy. Wood Sci. Technol. 2006, 40, 709-720.

38. Hart, J.F.; de Araujo, F.; Thomas, B.R.; Mansfield, S.D. Wood quality and growth characterization across intra-and inter-specific hybrid aspen clones. Forests 2013, 4, 786-807.

39. Thomas, S.C.; Martin, A.R. Carbon content of tree tissues: A synthesis. Forests 2012, 3, 332-352.

40. Johnsen, K.H.; Samuelson, L.J.; Sanchez, F.G.; Eaton, R.J. Soil carbon and nitrogen content and stabilization in mid-rotation, intensively managed sweetgum and loblolly pine stands. For. Ecol. Manag. 2013, 302, 144-153.

41. Samuelson, L.J.; Eberhardt, T.L.; Bartkowiak, S.M.; Johnsen, K.H. Relationships between climate, radial growth and wood properties of mature loblolly pine in hawaii and a northern and southern site in the southeastern united states. For. Ecol. Manag. 2013, 310, 786-795.

42. Jiang, W.; Han, G.; Via, B.K.; Tu, M.; Liu, W.; Fasina, O. Rapid assessment of coniferous biomass lignin-carbohydrates with near-infrared spectroscopy. Wood Sci. Technol. 2014, 48, $109-122$. 
43. Jones, P.D.; Schimleck, L.R.; Daniels, R.F.; Clark Iii, A.; Purnell, R.C. Comparison of pinus taeda 1. Whole-tree wood property calibrations using diffuse reflectance near infrared spectra obtained using a variety of sampling options. Wood Sci. Technol. 2008, 42, 385-400.

44. Jiang, W.; Han, G.; Zhang, Y.; Wang, M. Fast compositional analysis of ramie using near-infrared spectroscopy. Carbohydr. Polym. 2010, 81, 937-941.

45. Fang, Y.; Park, J.I.; Jeong, Y.-S.; Jeong, M.K.; Baek, S.H.; Cho, H.W. Enhanced predictions of wood properties using hybrid models of pcr and pls with high-dimensional nir spectral data. Ann. Oper. Res. 2011, 190, 3-15.

46. Malkavaara, P.; Alén, R. A spectroscopic method for determining lignin content of softwood and hardwood kraft pulps. Chemom. Intell. Lab. Syst. 1998, 44, 287-292.

47. Ferraz, A.; Baeza, J.; Rodriguez, J.; Freer, J. Estimating the chemical composition of biodegraded pine and eucalyptus wood by drift spectroscopy and multivariate analysis. Bioresour. Technol. 2000, 74, 201-212.

48. Sluiter, A.; Ruiz, R.; Scarlata, C.; Sluiter, J.; Templeton, D. Determination of extractives in biomass. Lab. Anal. Proced. (LAP) $\mathbf{2 0 0 5}, 1617$.

49. Sluiter, A.; Hames, B.; Ruiz, R.; Scarlata, C.; Sluiter, J.; Templeton, D.; Crocker, D. Determination of structural carbohydrates and lignin in biomass. Lab. Anal. Proced. 2008; pp. 1-15.

50. Thygesen, L.G.; Lundqvist, S.-O. NIR measurement of moisture content in wood under unstable temperature conditions. Part 2. Handling temperature fluctuations. J. Near Infrared Spectrosc. 2000, 8, 191-199.

51. Pedro, A.M.K.; Ferreira, M. Simultaneously calibrating solids, sugars and acidity of tomato products using PLS2 and NIR spectroscopy. Anal. Chim. Acta 2007, 595, 221-227.

52. Via, B.; Shupe, T.; Groom, L.; Stine, M.; So, C. Multivariate modelling of density, strength and stiffness from near infrared spectra for mature, juvenile and pith wood of longleaf pine (Pinus palustris). J. Near Infrared Spectrosc. 2003, 11, 365-378.

53. Via, B. Characterization and evaluation of wood strand composite load capacity with near infrared spectroscopy. Mater. Struct. 2013, 46, 1801-1810.

54. Via, B.; Eckhardt, L.; So, C.; Shupe, T.; Groom, L.; Stine, M. The response of visible/near infrared absorbance to wood-staining fungi. Wood Fiber Sci. 2006, 38, 717-726.

55. Via, B.; So, C.; Eckhard, L.; Shupe, T.; Groom, L.; Stine, M. Response of near infrared diffuse reflectance spectra to blue stain and wood age. J. Near Infrared Spectrosc. 2008, 16, 71-74.

56. Ryu, S.R.; Noda, I.; Lee, C.H.; Lee, P.H.; Hwang, H.; Jung, Y.M. Two-dimensional correlation analysis and waterfall plots for detecting positional fluctuations of spectral changes. Appl. Spectrosc. 2011, 65, 359-368.

(C) 2014 by the authors; licensee MDPI, Basel, Switzerland. This article is an open access article distributed under the terms and conditions of the Creative Commons Attribution license (http://creativecommons.org/licenses/by/3.0/). 